\title{
Reflets
}

Revue ontaroise d'intervention sociale et communautaire

\section{Réseau d'entraide : programme de formation personnelle auprès des femmes aînées}

\section{Johanne Pomerleau et Manon Lemonde}

Volume 1, numéro 2, automne 1995

La santé communautaire en Ontario français : défis et espoirs

URI : https://id.erudit.org/iderudit/026083ar

DOI : https://doi.org/10.7202/026083ar

Aller au sommaire du numéro

Éditeur(s)

Reflets : Revue ontaroise d'intervention sociale et communautaire

ISSN

1203-4576 (imprimé)

1712-8498 (numérique)

Découvrir la revue

Citer cet article

Pomerleau, J. \& Lemonde, M. (1995). Réseau d'entraide : programme de formation personnelle auprès des femmes aînées. Reflets, 1(2), 171-183. https://doi.org/10.7202/026083ar

Tous droits réservés (C) Reflets : Revue ontaroise d'intervention sociale et communautaire, 1995
Ce document est protégé par la loi sur le droit d'auteur. L'utilisation des services d'Érudit (y compris la reproduction) est assujettie à sa politique d'utilisation que vous pouvez consulter en ligne.

https://apropos.erudit.org/fr/usagers/politique-dutilisation/ 


\section{Réseau d'entraide:}

\section{programme de formation personnelle auprès des femmes aînées}

\section{Johanne Pomerleau}

Professeure adjointe, É cole des sciences infirmières, U niversité L aurentienne, Sudbury

\section{$M$ anon L emonde}

Professeure adjointe, É cole des sciences infirmières, U niversité L aurentienne, Sudbury

\section{Introduction}

Le présent article a pour but de présenter les méthodes d'évaluation de l'impact d'un programme de formation personnelle intitulé $D$ écouverte de soi auprès des femmes aînées francophones de la région de Sudbury. En plus de situer ce programme dans son contexte particulier qu'est le R éseau d'entraide, il sera question des écrits pertinents qui appuient ce genre d'intervention, des méthodes d'évaluation utilisées, des résultats obtenus et des conclusions qui peuvent être tirées de cette approche.

\section{Revue de la littérature}

Le réseau social peut être une source de soutien et un moyen de prévenir l'isolement social pour les ainés. Plusieurs recherches 
«L a solitude et

l'isolement social, voic des problèmes fréquemment véas par les femmes âgées, francophones ou autres 》 réalisées auprès de cette population confirment l'importance du réseau social et des ressources personnelles comme soutien permettant de préserver l'estime de soi et favorisant le maintien de la santé à cette étape de la vie. Les recherches n'ont pas réussi toutefois à démontrer la constance de cet effet bénéfique (M or-Barak, M iller et Syme, 1991). À ce jour, les études ne permettent pas d'identifier quel élément de l'intervention permet de maintenir la santé, compte tenu d'autres facteurs agissant tels que le statut fonctionnel et les problèmes reliés au vieillissement.

$\mathrm{H}$ unter, Linn et $\mathrm{H}$ arris (1982) ont pu conclure que les aînées qui demeurent seules ont en général une satisfaction face à la vie et une estime de soi diminuées, entrainnant ainsi chez elles un plus haut degré d'inadaptation sociale comparativement aux femmes qui vivent avec quelqu'un. Ce qui laisse croire que l'estime de soi et l'image de soi peuvent avoir un effet sur le bien-être physique et mental d'une personne. Tel que rapporté par W hall (1987), les personnes qui ont une faible estime de soi sont prédisposées à la maladie. De leur côté, Zémore et Bretell (1983) considèrent qu'une estime de soi diminuée conduit à la dépression et à l'isolement (Joshi, Garon et Lechasseur, 1984).

La solitude et l'isolement social, voici des problèmes fréquemment vécus par les femmes âgées, francophones ou autres. Cette situation est en partie reliée au processus de vieillissement normal (diminution de la mobilité et des capacités sensori-perceptuelles), mais aussi à la maladie, à la perte de son rôle dans la société ainsi qu'à la perte d'amis/ amies ou de membres de la famille suite au décès ou au déménagement. Tous ces facteurs contribuent au sentiment d'isolement social et de solitude. H étu (1992) affirme que «es différentes pertes reliées au vieillissement, de même que la tendance au désengagement chez plusieurs sujets âgés, sont de nature à exercer un effet de contraction sur le réseau de soutien» (p.64). M alheureusement, tous ces événements se produisent au moment où les aînés ont besoin de soutien pour réussir à s'adapter à leur nouvelle situation de vie et ainsi demeurer en santé autant sur les plans physique que psychologique. H étu (1992) a observé que les aînées possédant et utilisant un réseau social adéquat ont une meilleure santé physique 
«... la céation d'un réseau d' entraide pourrait sans doute aider les femmes aînées à contrecarrer les effets du vieillisse ment sur leur réseau.» et mentale. Selon Eliopoulos (1990), les aînées doivent, pour maintenir un état de bien-être, réaliser un équilibre satisfaisant entre les périodes de solitude et d'interaction sociale avec leur environnement.

Selon A ntonucci (1990), I'appartenance à un bon réseau amène le sujet à se percevoir positivement et donc à être davantage porté à mobiliser ses ressources pour faire face adéquatement aux exigences de la vie. Kessler et Essex (1982), Pearlin, Liberman, $M$ enaghan et al. (1981) soutiennent également que les moyens d'adaptation émotionnelle combinés à l'accessibilité des autres ressources personnelles et sociales préviendraient la dépression en maintenant une image de soi positive ou en aidant la personne à affronter et à surmonter ses difficultés.

Ces auteurs nous permettent d'affirmer que la création d'un réseau d'entraide pourrait sans doute aider les femmes aînées à contrecarrer les effets du vieillissement sur leur réseau social et à mettre en valeur leurs ressources personnelles.

\section{Survol du programme}

Actuellement, l'accent est mis sur les programmes de promotion de la santé afin de redonner aux ainées la confiance et le pouvoir d'entreprendre des démarches pour augmenter leur satisfaction personnelle tout en favorisant leur prise en charge personnelle. Le programme R éseau d'entraide de Samuels et Cole (1988), offert en français dans la région de Sudbury depuis 1991 répond à cet objectif, soit de promouvoir la capacité des aînées de s'aider et possiblement de s'entraider.

Trois volets composent le programme $R$ éseau d'entraide. Les personnes doivent tout d'abord s'inscrire au premier volet intitulé D écouverte de soi (DDS). Par la suite, certaines participantes ayant les aptitudes nécessaires et démontrant une capacité de diriger d'autres aînées, pourront passer au deuxième volet qui est la $F$ ormation d'animateurs communautaires (FAC). Enfin, les animatrices 
communautaires pourront s'inscrire au troisième volet, la $F$ ormation des coanimateurs communautaires (FCAC), qui les prépare à aider les aînées de leur communauté à compléter le volet $D$ écouverte de soi.

Tel que déjà mentionné, le projet décrit touche particulièrement la dispensation du premier volet D D s et son impact sur les femmes aînées.

Le volet D DS a pour but d'aider les aînées à reconnaître qu'elles sont encore capables d'apprendre, qu'elles ont d'importants acquis et qu'ils peuvent enrichir leur vie ainsi que celle des autres par le partage de leurs expériences. Ce programme de formation d'une durée de vingt heures leur permet de découvrir leur capacité de changer leur situation si ellesle désirent, nonobstant le fait qu'elles ont le choix d'effectuer tel changement dans leur vie personnelle.

\section{Survol des participantes}

A près avoir complété le volet DDS et afin de comprendre comment ce volet influence le réseau social des participantes, leur santé mentale et leur estime de soi, nous avons demandé à un groupe de 43 femmes francophones âgées de 56 à 75 ans de participer à notre projet. D eux seuls critères d'admissibilité ont été retenus pour participer au projet d'évaluation, soit d'être âgée de 50 ans et plus et d'avoir terminé le volet DDS.

D ans ce groupe, $47 \%$ des participantes étaient mariées, $35 \%$ étaient veuves, $2 \%$ étaient divorcées et $16 \%$ n'avaient pas répondu à cette question. Elles avaient de deux à dix-sept enfants et $33 \%$ d'entre elles possédaient une scolarité de niveau secondaire. C ertaines d'entre elles demeuraient dans une résidence où elles avaient accès à des services de santé sur place (Tableau 1).

II n'existe pas d'explication à l'absence de réponses aux questions socio-démographiques, puisque les participantes étaient invitées à compléter personnellement ce questionnaire. 
Tableau 1 - C aractéristiques socio- démographiques des participantes

\begin{tabular}{ccc}
\hline Âge & $\mathbf{N}=\mathbf{4 3}$ & $\mathbf{\%}$ \\
$40-45$ & 1 & 2 \\
$46-50$ & 2 & 5 \\
$51-55$ & 6 & 14 \\
$56-60$ & 7 & 16 \\
$61-65$ & 7 & 16 \\
$66-70$ & 4 & 9 \\
$71-75$ & 9 & 21 \\
Sans réponse & $\mathbf{7}$ & $\mathbf{1 6}$ \\
& & \\
État civil & $\mathbf{N}$ & $\mathbf{4}$ \\
mariée & 20 & 47 \\
veuve & 15 & 35 \\
divorcée & 1 & 2 \\
Sans réponse & $\mathbf{7}$ & $\mathbf{1 6}$ \\
& & \\
Scolarité & $\mathbf{N}$ & $\mathbf{2}$ \\
primaire & 10 & 33 \\
secondaire & 14 & 16 \\
post-secondaire & 7 & $\mathbf{2 8}$ \\
Sans réponse & $\mathbf{1 2}$ &
\end{tabular}

\section{Méthode d'évaluation}

Les instruments de mesure sélectionnés devaient nous permettre de comprendre comment ce programme de formation influence les ainées. Les instruments de mesure utilisés sont les suivants:

a) la version française du $D$ epressive $A$ djective $C$ hedk $L$ ist (DACL-E) (Lubin, 1981) pour évaluer les perceptions que les aînées ont face à leur santé mentale;

b) la version française du C ulture F ree Self-E steem Inventory for A dults (CFSEI-AD) (Battle, 1992) pour évaluer l'estime de soi; 
c) la traduction et l'adaptation française du L ubben Sodal N etwork Scale (LSN S) (Lubben, 1988) et le diagramme de C urtis (1979) pour mesurer l'ampleur du réseau social. C es questionnaires ont été complétés à la fin du volet DDS.

Le DACL a été développé par Lubin (1981) pour mesurer l'humeur dépressive. La version $\mathrm{E}$ de cet instrument est spécifique aux personnes aînées et peut être complétée en deux minutes par des sujets possédant une scolarité de niveau primaire. Les participantesidentifient, à partir d'une liste de 34 adjectifs non répétitifs, tous les adjectifs qui les décrivent de façon générale.

LE CFSEI-AD de Battle (1992) permet de mesurer la perception qu'une personne a de sa propre valeur. L'instrument de mesure comprend 40 points pour mesurer l'estime de soi. Les réponses peuvent être regroupées en quatre sous-catégories: l'estime de soi générale, sociale, personnelle et l'attitude défensive en évaluant l'usage du mensonge.

Le LSN s développé par Lubben (1988) a pour but de déterminer la grandeur du réseau social actif des participantes et la fréquence des interactions avec les différents membres du réseau. $C$ et instrument permet d'identifier les personnesqui sont isolées, en évaluant les relations avec les amis/ amies, les membres de la famille et les relations de confiance ou de soutien.

Le diagramme composé de cercles concentriques de $C$ urtis permet de visualiser de quelles façons les participantes représentent sur papier la distribution de leur réseau social (famille, amis) amies, voisins/ voisines), ce qui nouspermet de déterminer approximativement le nombre de personnes qui composent le réseau de chacune des participantes. Les informations ainsi recueillies ne permettent pas d'évaluer la qualité des relations ni les bénéfices que les aînées peuvent en retirer.

À la fin du volet DDS, avec l'animatrice, nous avons expliqué aux 43 ainées le but du projet et la procédure utilisée pour évaluer l'impact du programme de formation. Trente-deux d'entre elles ont accepté de compléter individuellement les quatre ins truments de mesure. $\mathrm{N}$ ous sommes toutefois demeurées à leur disposition pendant qu'elles complétaient les outils de mesure afin de répondre à leurs questions. 


\section{Résultats et interprétation}

M ême si les 32 femmes disaient se sentir mieux, seulement deux d'entre elles (6,3\%) ont choisi l'adjectif positif «bien dans sa peau» du DAC L-E (Tableau 2) à la fin du volet D DS.

Tableau 2 - F réquences représentant les choix d' adjectifs positifs par les participantes (DACL-E)

\begin{tabular}{lcc}
\hline $\begin{array}{c}\text { 12 adjectifs positifs } \\
\text { du DACL-E }\end{array}$ & $\mathbf{N = 3 2}$ & \% \\
\hline bien dans sa peau & 2 & 6,3 \\
paisible & 16 & 50,0 \\
en forme & 18 & 56,3 \\
calme & 18 & 56,3 \\
vigoureuse & 18 & 56,3 \\
libre & 20 & 62,5 \\
forte & 22 & 68,8 \\
en sécurité & 22 & 66,8 \\
bien & 23 & 71,9 \\
chanceuse & 23 & 71,9 \\
active & 24 & 75,0 \\
de bonne humeur & 26 & 81,3 \\
\hline
\end{tabular}

«Bien dans sa peau» est le seul adjectif positif qui a été choisi aussi peu fréquemment; les autres adjectifs positifs ont une fréquence relative de $50 \%$ et plus, tandis que l'adjectif négatif «se sentir seule» a une fréquence relative de $40,6 \%$ (Tableau 3). C es observations peuvent s'expliquer si I'on considère que $31,2 \%$ (12 sur 32) des femmes demeurent seules et que $35 \%$ sont veuves (15 sur 43). Plusieurs autres facteurs doivent également être considérés, dont la prise de conscience qu'elles ont faite en participant à ce programme, l'âge, les changements physiologiques reliés au vieillissement, la définition subjective accordée aux expressions du DACL-E, le niveau personnel d'estime de soi et enfin le fait qu'il s'agissait de la dernière rencontre du groupe. C es résultats pourraient 
laisser entrevoir un problème.Toutefois, il ne faut pas oublier que seulement une personne dans le groupe, selon le DACL-E, serait d'humeur dépressive, donc à risque.

Tableau 3 - F réquences représentant les choix d' adjectifs négatifs par les participantes (DAC;-E)

\begin{tabular}{lcc}
\hline $\begin{array}{c}\text { 22 adjectifs négatifs } \\
\text { du DACL-E }\end{array}$ & $\mathbf{N}=\mathbf{3 2}$ & $\mathbf{\%}$ \\
\hline affreuse & 0 & 0 \\
apathique & 1 & 3,1 \\
malheureuse & 1 & 3,1 \\
au bout du rouleau & 2 & 6,3 \\
délaissée & 2 & 6,3 \\
torturée & 2 & 6,3 \\
maussade & 2 & 6,3 \\
sans espoir & 2 & 6,3 \\
perdue & 3 & 9,4 \\
abattue & 3 & 9,4 \\
indifférente & 4 & 12,5 \\
triste & 5 & 15,6 \\
démoralisée & 5 & 15,6 \\
avoir les bleus, démotivée & 6 & 18,8 \\
critiquée & 7 & 21,9 \\
angoissée & 7 & 21,9 \\
affaissée & 7 & 21,9 \\
enchaînée & 7 & 21,9 \\
brisée & 8 & 25,0 \\
accablée & 9 & 28,1 \\
se sentir seule & 13 & 40,6 \\
\hline
\end{tabular}

II faut également noter que ces deux expressions, «bien dans sa peau» et «se sentir seule», reviennent à plusieurs reprises au sein du volet D DS. En effet, ces aînées ont discuté des aspects sains du vieillissement, en échangeant sur leurs craintes ou sur leurs forces et en mettant en pratique des techniques de communication. De cette façon, elles ont appris à découvrir les autres et réalisé que les membres du groupe peuvent agir en tant que source de soutien. 
«... 40,6\% de ces femmes sont rarement ou seulement quelque fois consultées par d'autres personnes qui ont des décisions importantes à prendre»
L'animatrice a un rôle important à jouer, car elle doit dès le début offrir le soutien et les encouragements nécessaires au progrès des membres du groupe. Son rôle est non seulement relié à la direction du groupe, mais elle agit aussi comme modèle.

$\mathrm{N}$ ous avons cherché à identifier dans l'échelle qui mesure le réseau social (LSNS) et l'estime de soi (CFSEI-AD) ces deux mêmes expressions, soit «bien dans sa peau» et se «sentir seule».

Tout d'abord, les scores du LSN S aux trois questions qui permettent d'évaluer le réseau familial démontrent que $90,6 \%$ des aînées ont 5 entretiens ou plus avec des membres de leur famille et que $78,2 \%$ d'entre elles en ont quelques fois par semaine ou même tous les jours. Beaucoup de personnes semblent entourer ces femmes. Par contre, d'autres résultats démontrent que 28,2 \% ont de 0 à deux échanges avec des amis/amies par mois. De plus, toutes ces femmes sont proches de deux membres ou plus de leur famille et ont au moins une confidente ou un confident. Toutefois, $31,2 \%$ ont rarement ou quelquefois quelqu'un de disponible avec qui elles peuvent discuter lorsqu'une décision doit être prise, alors que $40,6 \%$ de ces femmes sont rarement ou seulement quelquefois consultées par d'autres personnes qui ont des décisions importantes à prendre. Enfin, $40 \%$ des participantes n'ont personne qui compte sur elles pour accomplir une activité quelconque. Les scorestotaux bruts des participantes démontrent que $90,6 \%$ d'entre elles ont un réseau adéquat et qu'une seule femme souffrirait d'isolement social; ce qui ne coïncide pas très bien avec le fait que 40,6 \% de ces mêmes ainées aient choisi I'adjectif négatif «se sentir seule» du D ACL-E.

Le Tableau 4 présente cinq questions tirées du CFSEI-AD qui permettent d'explorer davantage comment les participantes définissent «se sentir seule». Plus de 40,6\% d'entre elles affirment avoir quelques amis/ amies, être seules la plupart du temps, être facilement déprimées, avoir de la difficulté à rencontrer de nouvelles personnes et avoir un manque d'initiative. Tous ces facteurs peuvent ainsi contribuer au sentiment de use sentir seule» exprimé par ces femmes. 
Tableau 4 - Q uestions permettant d' explorer comment les partidipantes définissent «se sentir seule»

\section{Q uestions du CFSEI-AD}

1. Avez-vous seulement quelques ami-e-s?

5. Passez-vous la plupart de votre temps libre seule?

12. Êtes-vous facilement déprimée?

22. Vous est-il difficile de rencontrer de nouvelles personnes?

39. Avez-vous définitivement un manque d'initiative?
$\mathbf{N}=32$

17

22

68,8

$17 \quad 53,1$

13

40,6

40,6

En ce qui concerne le score total dU CFSEI-AD , 53,13\% des participantes ont une estime de soi qui varie entre satisfaisante et très élevée à la fin du programme de formation, alors que $46 \%$ des autres ont obtenu un score représentant une estime de soi qui varie d'intermédiaire à basse.

Tableau 5 - R eprésentation des choix des participantes pour chaque niveau du réseau sodial

\begin{tabular}{lcccc}
\hline & $\mathbf{1}$ & $\mathbf{2}$ & $\mathbf{3}$ & $\begin{array}{c}\mathbf{4} \\
\text { (éloignée de moi) }\end{array}$ \\
& $97,3 \%$ & $37,8 \%$ & $16,2 \%$ & $8,1 \%$ \\
famille & $94,6 \%$ & $18,9 \%$ & $2,7 \%$ & $2,0 \%$ \\
$\begin{array}{l}\text { confident(s)/ } \\
\text { confidente(s) }\end{array}$ & & & & \\
$\begin{array}{l}\text { voisin(s) / } \\
\text { voisine(s) }\end{array}$ & $62,2 \%$ & $21,6 \%$ & $16,2 \%$ & $13,5 \%$ \\
$\begin{array}{l}\text { ami(s)/ } \\
\text { amie(s) }\end{array}$ & $78,4 \%$ & $40,5 \%$ & $10,8 \%$ & $5,4 \%$ \\
\hline
\end{tabular}

LeTableau 5 est la représentation graphique des cercles concentriques, les pourcentages représentant le nombre de personnes que les participantes du groupe ont identifié danschaque catégorie. Au 
total, $97,3 \%$ des participantes identifient une personne de leur famille comme étant proche d'elles; plus les niveaux s'éloignent d'elles, plus les pourcentages diminuent ( $8,1 \%$ au niveau 4$)$. C ette représentation leur permet d'identifier autant de personnesqu'elles le veulent et ce, selon différents niveaux de proximité.

\section{Conclusion et recommandations}

Il est évident que les résultats quantitatifs obtenus ne correspondent pas aux commentaires des participantes. Toutefois, nous ne pouvons pas nier qu'elles semblent se sentir mieux et disent se sentir mieux à qui veut bien les entendre. $D$ 'autres groupes qui ont suivi le même programme de formation, sans avoir fait partie du projet d'évaluation, ont exprimé des commentaires similaires. En effet, la majorité d'entre elles disent se sentir mieux et plus fortes que lorsqu'elles se sont inscrites au programme, d'autres disent avoir appris à exprimer leurs sentiments et avoir réalisé leur valeur personnelle aux yeux des autres. U ne participante décrit son apprentissage ainsi : «Pour moi, ça m'a beaucoup aidée à ouvrir mes yeux, à accepter ma situation alors que je me sentais seule et déprimée. A ujourd'hui, je pars avec beaucoup plus d'enthousiasme et d'encouragement. Je me sens beaucoup plus forte et plus heureuse.»

Présentement, il nous est impossible de répondre à la question suivante: «Pourquoi, à la fin du programme D DS, ces femmes disent se sentir mieux et plus fortes que lorsqu'elles ont débuté le programme?» Les résultats recueillis à l'aide des questionnaires démontrent que seulement quelques-unes d'entre elles se sentaient bien dansleur peau même si toutes avaient un réseau social assez élaboré, c'est-à-dire que malgré la présence d'un réseau composé de plusieurs personnes, elles se sentaient quand même seules. II est important de noter qu'une seule personne a répondu qu'elle avait rarement quelqu'un pour discuter de problèmes alors que $53,1 \%$ ont très souvent ou toujours quelqu'un qui est 
prêt à les écouter. Toutefois, 40,6 \% de ces femmes étaient rarement consultées lorsque d'autres personnes ont des décisions importantes à prendre et ce, même si 68,8 \% d'entre elles disaient que quelqu'un compte sur elles à tous les jours.

A près la dernière session du volet $D D S$, l'animatrice a observé que les participantes semblent mettre en pratique les apprentissages réalisés au cours du programme de formation, surtout les techniques de communication.

II n'est pas facile d'évaluer l'impact d'une intervention en promotion de la santé, faute d'outil mesurant spécifiquement les effets de l'intervention. La plus grande difficulté réside dans le choix et l'identification de la ou des variables à mesurer. $\mathrm{N}$ ous croyons encore que ce programme de formation a un impact positif auprès des aînées, parce que les commentaires qu'elles émettent se répètent d'un groupe à l'autre. Les résultats obtenus nous laissent croire qu'il faudra utiliser une approche davantage qualitative pour arriver à comprendre ce que ces femmes expriment en répondant aux questions suivantes:

1. Pourquoi les femmes aînées, après avoir complété le programme de formation, disent se sentir mieux et plus fortes?

2. Est-ce le programme de formation ou la dynamique de groupe qui influence le plus les participantes?

3. Est-ce que ce sentiment de solitude et de force persiste ou est-il éphémère après la dissolution du groupe?

II ne serait possible de répondre à ces questions qu'à partir d'une étude longitudinale qui utiliserait des méthodes de recherche qualitative et quantitative afin d'évaluer les effets d'un programme en promotion de la santé sur les aînées qui y participent. L'approche pourrait alors être composée de pré-tests, de post-tests et d'un suivi 6 à 12 mois après la fin du volet D DS.

\section{Bibliographie}

AN TO N U C CI, T. (1990). «Social supports and social relationships» dans R . Binstock et L. George (Eds). $\mathrm{H}$ andbook of $\mathrm{A}$ ging and the Social Sciences. $\mathrm{N}$ ew York: A cademic Press.

BAT TLE, J. (1992). C ulture-F ree Self-E steem Inventories (2nd ed.). A ustin, Texas: Pro-ed.

BIEGEL, D.E., SH OR E, B.K. et GOR DON , E. (1984). B uilding Support N etworks for the Elderly, Theory and A pplications. Beverly H ills, CA: Sage Publications. 
ELIO PO U LO S, C. (1993).G erontological N ursing (3rd Ed). Philadelphia, Pennsylvania; J.B. Lippincott. HÉTU, J.-L. (1992). Psychologie du vieillissement. M ontréal: Éditions du M éridien.

KESSLER , R . et ESSEX, M .J. (1982). «M arital Status and D epression:T he Importance of C oping R esources). Social Forces, 61, 481-507.

LU BBEN , J.E. (1988). «Assessing Social N etworksAmong Elderly Populations». Family C ommunity H ealth, 11(3), 42-52.

LU BIN , B. (1981). D epression A djective C heck L ist: M anuel. San Diego, C A: Educational and Industrial Testing Service.

M OR -BAR AK, M .E. et M ILLER , L.S. (1991). «A Longitudinal Study of the C ausal R elationship Between Social $N$ etworks and $\mathrm{H}$ ealth of the Poor Frail Elderly». Journal of A pplied $\mathrm{G}$ erontology, 10(2), 77-84.

PEAR LIN, L.I., LIBER M AN, E.G., MENAGHAN, M.A. et MULLAN, J.T. (1981). «T he Stress Processs. Journal of $H$ ealth and Sodial B ehavior, 22(4), 337-356.

SAM U ELS, S. et CO LE, P. (1988). L e réseau d'entraide des personnes âgées.

TO SH I, P., GARO N , L. \& LECH ASSEU R, S. (1984). «Self-Esteem and Loneliness A mong U nemployed Women». P sychological R eports, 54, 903-906.

W H ALL,A.L. (1987). «Self-E steem and the M ental H ealth of O Ider A dults». Journal of G erontological N ursing, 13(4), 41-42.

ZEM OR E, R . \& BR ET ELL, D. (1983). «D epression-Proneness, Low Self-Esteem, U nhappy O utlook and N arcissistic Vulnerability». P sychological R eports, 52, 223- 230. 